\title{
Research and Development of a Digital Safety Monitoring Device for Attached Lifting Scaffolding
}

\author{
Zhang Qiang,** \\ ${ }^{1}$ Guangdong Construction Vocational Technology Institute, Guangzhou, Guangdong. 510440, China
}

\begin{abstract}
This paper introduces a digital safety monitoring device for attached lifting scaffold, including attached lifting scaffold and safety monitoring control device. The safety monitoring device includes monitoring point, intermediate station, control center, communication converter, display and mobile terminal. The monitoring point is set on the attached lifting scaffold, the monitoring point is wirelessly connected with the intermediate station. The intermediate station transmits data to the control center through network mapping. The control center is respectively connected with the display and the communication converter, and the communication converter is connected with the mobile terminal. The utility model has simple structure, convenient operation, can intuitively monitor various values of the monitoring points, and provides a strong guarantee for the construction safety of the attached lifting scaffold.
\end{abstract}

\section{Introduction}

Attached lifting scaffolding refers to the external scaffold, which is erected with a certain height and attached to the engineering structure, and can climb or descend layer by layer with the engineering structure by relying on its own lifting equipment and devices. In JGJ 202-2010 "Technical Specification for Safety of Construction lmplementary Scaffolding", it is required to have the function of prevent overturn and prevent falling.

At present, there are many kinds of attached lifting scaffolding, and it is still in a period of rapid development. There is no unified standard in the industry, whether it is production and design, or classification. According to the classification of frame performance, the attached lifting scaffold can be divided into ordinary type and all steel type. All steel attached lifting scaffold is widely used in office buildings with regular shape and some municipal projects, while ordinary attached lifting scaffold is often used in middle and low rise civil buildings. According to the classification of major vertical frame form, the attached lifting scaffold can be divided into single piece type and space truss type. Because of the advantages of single piece type, such as simple design, low cost, material saving, convenient transportation, and saving man-hours in installation, it is widely used at present. The space truss type has great rigidity, but due to the higher cost, it is currently used less. According to the classification of lifting system, the attached lifting scaffold can be divided into electric hoist type, hydraulic type, rack and pinion type, worm and turbine type, etc. At present, the commonly used types are electric hoist type and hydraulic type. In addition, the attached lifting scaffold can also be classified according to the force on the frame body during lifting and the way of unloading.

\section{Features of attached lifting scaffold}

Compared with the traditional scaffold, the attached lifting scaffold has the characteristics of low carbon, safety, industrialization and intelligence. After nearly 30 years of development, it has been widely used in the construction of high-rise and super high-rise buildings. It can be predicted that the attached lifting scaffolding will play an increasingly important role in the future. Specifically, the attached lifting scaffold has the following characteristics:

-In line with the development direction of industrial production.

With the appearance of attached lifting scaffold, the original scattered, complicated and high-risk manual operation is replaced by mechanical equipment. It is assembled at the bottom of the main building at one time and attached to the building. With the increase of floor height, it is continuously improved, and the construction mechanization of scaffold operation has been realized.

-Low energy consumption.

Green environmental protection. Steel consumption reduced by $70 \%$, power consumption reduced by $90 \%$, construction consumables saved by $30 \%$.

-Outstanding economic indicators.

It is suitable for high-rise buildings, especially super high-rise buildings. The higher the floor, the more economical it is, and the average cost can be reduced by $30 \%-60 \%$

- Wide application.

All kinds of structure of the main body of the building are applicable.

- High security.

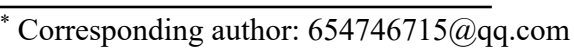


Reduce the fire hazards caused by scaffold burning, reduce the proportion of building safety accidents caused by high-risk operations such as falling from height and object strike, and facilitate safety production inspection.

- High degree of intelligence.

Due to the use of digital technology control system, it can display the lifting status in real time and automatically collect the load value of each lifting position.

- Improve the level of civilized construction.

Compared with the confusion of traditional scaffold appearance, the attached lifting scaffold is not landing, and the construction operation layer can be closed, so as to make the overall construction project regular and orderly, which is conducive to display the safety and civilized image of the construction project.

\section{Accident prevention of attached lifting scaffold}

As the attached lifting scaffolding is a dangerous temporary construction facility, a series of problems will still occur in the process of use, resulting in serious security risks. For example, in 2011, a high-rise attached scaffold falling accident occurred at the construction site of Kaixuan building in Xi'an City, Shaanxi Province, causing 10 deaths and 2 serious injuries. The relevant construction management procedures of the project are not complete. The cause of the accident was that the construction unit did not evacuate the operators on the scaffold when implementing the overall lifting of the attached scaffold, and illegally removed the load-bearing components, resulting in the unbalanced fall of the frame. In March 2019, in the new cable project of Baosheng Ocean Engineering in Yangzhou Economic and Technological Development Zone, the attached lifting scaffold suddenly lost its stability and fell from a high place (about 16 floors) to the landing scaffold of 1-6 floors, resulting in 7 workers' deaths. The direct cause of the accident is that the construction unit uses steel wire rope to replace the climbing frame lifting support in violation of regulations, manually removes all anti falling devices and anti overturning devices of the climbing frame, and unplugs the signal line of the synchronous control device. When the load near the lifting point of the frame increases, causing local damage, the frame loses overload protection and shutdown function, resulting in chain reaction, resulting in the overall fall of the frame.

At present, the common problems in the construction of the attached lifting scaffold mainly include that the prevent overturning device is not in place, the components are not accepted when material entrance, the connecting wall parts are less, the scaffold is not set up in strict accordance with the special design scheme, the unconventional demolition of the scaffold and so on.

In order to prevent accidents of attached lifting scaffolds, on the one hand, we must make corresponding technical countermeasures (design according to the specification requirements, equip with synchronous control system, prevent falling device, prevent overturning device and other security devices, etc.) and management countermeasures (establish standardized safety management system, improve enterprise technology and management level, do a good job in safety disclosure, and implement safety responsibilities, etc.), On the other hand, it is necessary to upgrade the safety monitoring system.

\section{Attached lifting scaffold digital safety monitoring device}

\subsection{Technical background}

At present, the methods of monitoring the balance state of the whole lifting scaffold during the lifting process can be divided into two categories: manual monitoring and computer monitoring. In the manual monitoring method, the influence of human factors is very large. In the engineering application, the construction personnel can only find the visual situation such as attached lifting scaffold body lifting obstacles and machine failure with the naked eye, they can not find the hidden danger such as asynchronous lifting, overload and so on. With the improvement of the safety requirements and the degree of automation, this method will gradually be replaced by the computer real-time monitoring method. However, in the integral lifting scaffolding computer monitoring system that has been applied, almost all adopt centralized monitoring methods. This kind of monitoring system has many defects, such as a large amount of cables used, poor anti-interference ability in the transmission process, resulting in inaccurate measurement values, and unintuitive display methods.

According to JGJ202-2010 "Technical Specification for Safety of Construction lmplementary Scaffolding", when the attached lifting scaffold is raised and lowered, it must be equipped with a synchronous control system that limits the load or level difference. The continuous horizontal support truss should adopt the limited load automatic control system; the simply supported static horizontal truss should adopt the horizontal elevation difference synchronous automatic control system; if the equipment is limited, the limited load automatic control system can be selected. The limited load automatic control system shall have the following functions: (1) when the load of a certain machine position exceeds $15 \%$ of the design value, it shall automatically alarm and display the alarm position in the form of sound and light, and when it exceeds $30 \%$, it shall be able to stop the lifting equipment automatically. (2) It shall have the functions of overload, loss of load, alarm and shutdown. It is advisable to add display memory and storage functions. (3) In addition to have its own fault alarm function, it shall also adapt to the on-site environment. (4) The performance should be reliable and stable, and the control accuracy should be within $5 \%$. The horizontal height difference synchronous control system shall have the following functions: (1) when the height difference between the two ends of the horizontal support truss reaches $30 \mathrm{~mm}$, it shall be able to stop automatically, and the reason should be investigated and adjusted before lifting. (2) It shall be able to display the actual elevation and superelevation data of 
each lifting point, and have the function of memory and storage.

Although the existing attached lifting scaffold load pre-warning safety protection system can display the overload position and give an alarm, it can not meet the requirements of "display and record the position load value and displacement value" proposed by the current specification. Therefore, it is necessary to develop a digital safety monitoring system for attached lifting scaffold that can display and control the position load and displacement value, so as to provide reliable measures for the safe use of the attached lifting scaffold.

\subsection{Technical content}

\subsubsection{Problems to be solved}

The research and development device described in this article belongs to the field of safety control technology, and relates to a safety monitoring device for detecting the attached lifting scaffold through the Buck equivalent circuit, and a method for real-time observing the machine position load, lifting amount and other data of each machine position of the scaffold through the safety monitoring device. The problems existing in the prior art are solved.

(1) The amount of cables used is large and the construction is inconvenient;

(2) Using cable to transmit analog signal directly has poor anti-interference ability, which affects the accuracy of measured value;

(3) The digital form of display is not intuitive, so it is difficult for the operator to make a comprehensive estimate of the balance degree of the scaffold before a certain measured value exceeds the limit.

(4) Data monitoring mainly relies on people's passive attention and lacks active alarm and interactive mode.

\subsubsection{Technical scheme}

The technical solution adopted by the equipment described in this article is to provide a digital safety monitoring device for attached lifting scaffold with simple structure, convenient operation and intuitive observation of various values of monitoring points in view of the above-mentioned problems in the prior art.

The utility model is realized through the following technical scheme:

A digital safety monitoring device for attached lifting scaffold, including attached lifting scaffold and safety monitoring control device. The safety monitoring device includes monitoring point, intermediate station, control center, communication converter, display and mobile terminal. The monitoring point is set on the attached lifting scaffold, the monitoring point is wirelessly connected with the intermediate station. The intermediate station transmits data to the control center through network mapping. The control center is respectively connected with the display and the communication converter, and the communication converter is connected with the mobile terminal. The communication converter is connected with the alarm device. The monitoring point is composed of a load sensor, a displacement sensor, a lifting displacement sensor and a Buck circuit. The intermediate station is provided with a differential coil. Figure 1 shows the attached lifting scaffold; Figure 2 is a workflow diagram.

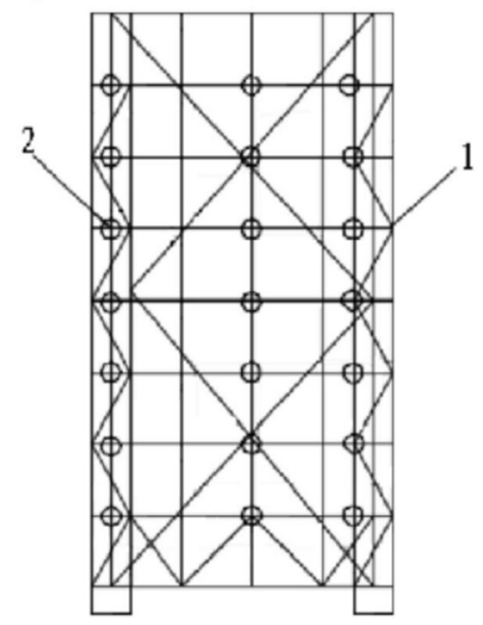

Figure 1. Attached lifting scaffold

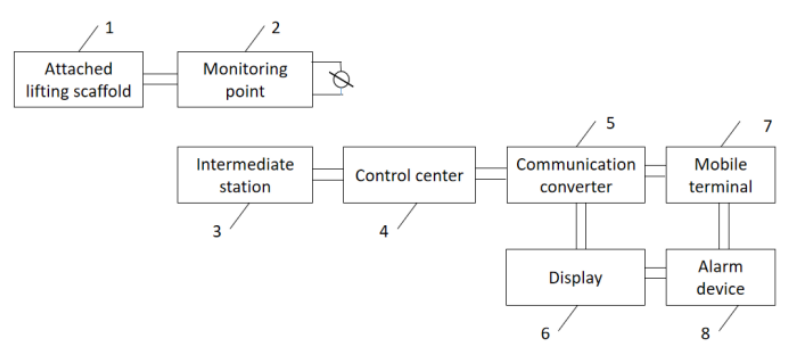

Figure 2. Workflow diagram

\subsection{Specific implementation mode}

In order to make the purpose, technical scheme and advantages of the utility model patent more clear, the following is a further detailed description of the utility model patent in combination with the drawings and examples.

\subsubsection{Component function description}

As shown in Figures 1 to 2, a digital safety monitoring device for attached lifting scaffold 1 includes attached lifting scaffold 1 and safety monitoring control device. The safety monitoring device includes monitoring point 2 , intermediate station 3 , control center 4 , communication converter 5 , display 6 , mobile terminal 7 and alarm device 8 . The monitoring point is set on attached lifting scaffold 1 , and the monitoring point 2 is wirelessly connected with intermediate station 3 , The intermediate station 3 transmits data to the control center 4 through the network mapping, The control center 4 is respectively connected with the communication converter 5 and the display 6 , and the communication converter 5 is respectively connected with the mobile terminal 7 and the alarm device 8 .

Among them, attached lifting scaffold 1 is mainly composed of attached lifting scaffold structure, attached 
support, prevent overturning device, prevent falling device, lifting mechanism and control device.

Monitoring point 2: it is responsible for measuring the load, displacement, lifting amount and other parameters of scaffold 1, and transmitting the data to intermediate station 3 through wireless transmission; monitoring point 2 is mainly composed of load sensor, displacement sensor, lifting displacement sensor and Buck circuit, The signal is monitored by the sensor, and the signal is sent to the Buck circuit for amplification to improve the accuracy of the signal observation.

Intermediate station 3: used to collect and summarize signals sent from monitoring point 2 .

Control center 4: mainly composed of industrial control single-chip microcomputer, responsible for data threshold analysis, classification, and control the next step of the action process.

Communication Converter 5: convert electronic signal into digital information through network architecture.

Display 6: it is mainly used to present the column chart of monitoring data and observe the threshold range of various indicators.

Mobile terminal 7: client terminal, which is used for the client's mobile device to log in and view the parameter information.

Alarm device 8: mainly placed in the construction site, used to generate audio when it exceeds or is about to exceed the parameter threshold, and send warning information to the construction personnel.

\subsubsection{Implementation examples}

The power supply of the utility model provides the power quality for the monitoring point 2 . The monitoring point 2 monitors and collects the relevant parameters of the attached lifting scaffold 1 through the load sensor, the displacement sensor and the lifting displacement sensor. After the parameters are detected, they are amplified by the Buck circuit to avoid the distortion of the output signal. After amplifying the signal, the data is transmitted to the intermediate station 3 . The intermediate station 3 collects the information sent from the monitoring point 2 for summary and converts the signal into voltage parameters through the differential coil; the voltage parameters are transmitted to the control center 4 through the network mapping; the control center 4 processes and analyzes the data information through the industrial single-chip microcomputer; if the parameters are within the reasonable threshold, the communication converter will not be started, and the calculation results will be directly displayed to the display 6 of the monitoring room; If the monitoring data reaches or exceeds the limited threshold, and the information is transmitted to the communication converter 5 , the communication converter 5 converts the information into digital information on the one hand, and sends it to the construction project manager, chief engineer and other relevant persons; at the same time, the alarm device 8 on the construction site is closed, the emergency safety warning information is played, and evacuates people to leave.
The above-mentioned embodiments and other illustrations only represent one embodiment of the utility model, and are only intended to illustrate rather than limit the present utility model. For ordinary technicians in the art, a number of modifications and improvements can be made without departing from the concept of the utility model.

\section{Summary}

The beneficial effect of the invention are

(1) It is possible to monitor the tiny load caused by the asynchronous lifting volume of multi-stations through the Buck circuit.

(2) Non-cable direct output signal, using an amplifier circuit to fidelity restore the monitored voltage and waveform.

(3) The safety monitoring system adopts a bar graph mode to reflect the parameters of each machine position, and the values of the monitoring points can be observed intuitively, and it is clear what position is currently at the threshold.

(4) There are fewer measurement points, reducing the amount of cables used and reducing construction pressure.

(5) With the help of the network platform protocol, an active early warning system is provided through mobile terminals to pre-warn the risks caused by load changes.

Through research and development, the digital safety monitoring device of the attached lifting scaffold has been greatly improved compared with the existing devices of the same type. It has a simple structure, simple and fast operation, and can accurately and intuitively monitor the values of the monitoring points. Through the implementation of automation, intelligence, and digitization, it provides a strong equipment guarantee for the construction safety of attached lifting scaffolding.

\section{References}

1. Wang, X.Q. (2013) Safety Analysis and Accident Prevention Countermeasures of Attached Lifting Scaffold System. Hubei Civil Construction, 42:288292.

2. Li, M. (2014) Research of attachment lifting scaffold's digital safety monitoring system. Construction Mechanization, 35(z1): 19-24.

3. Wang, F., WEN, X.B. (2016) The types and features of the commonly used adhesive lifting scaffolding. Construction Mechanization, 37(6):28-30

4. Du, R.J. (2000) Safety Design of Attached Vertically Moving Scaffold. Architecture Technology, 31(8): 520-522.

5. Zhou, F.X., (2015) Technical Innovation of Domestic Scaffold. Architecture Technology, 46(8):678-682.

6. Wei, M., (2017) The Climbing Scaffolding Design and Construction Technology of Ethiopia Union Bank Complex Building. Construction Technology, 46(16):72-75. 